\title{
A!
}

This is an electronic reprint of the original article.

This reprint may differ from the original in pagination and typographic detail.

Suomela, S.; Kutvonen, A.; Ala-Nissilä, Tapio

\section{Quantum jump model for a system with a finite-size environment}

Published in:

Physical Review E

DOI:

10.1103/PhysRevE.93.062106

Published: 03/06/2016

Document Version

Publisher's PDF, also known as Version of record

Please cite the original version:

Suomela, S., Kutvonen, A., \& Ala-Nissilä, T. (2016). Quantum jump model for a system with a finite-size environment. Physical Review E, 93(6), [062106]. https://doi.org/10.1103/PhysRevE.93.062106

This material is protected by copyright and other intellectual property rights, and duplication or sale of all or part of any of the repository collections is not permitted, except that material may be duplicated by you for your research use or educational purposes in electronic or print form. You must obtain permission for any other use. Electronic or print copies may not be offered, whether for sale or otherwise to anyone who is not an authorised user. 


\title{
Quantum jump model for a system with a finite-size environment
}

\author{
S. Suomela, ${ }^{1}$ A. Kutvonen, ${ }^{1}$ and T. Ala-Nissila ${ }^{1,2}$ \\ ${ }^{1}$ Department of Applied Physics and COMP Centre of Excellence, Aalto University School of Science, \\ P.O. Box 11100, 00076 Aalto, Finland \\ ${ }^{2}$ Department of Physics, P.O. Box 1843, Brown University, Providence, Rhode Island 02912-1843, USA
}

(Received 13 January 2016; revised manuscript received 14 March 2016; published 3 June 2016)

\begin{abstract}
Measuring the thermodynamic properties of open quantum systems poses a major challenge. A calorimetric detection has been proposed as a feasible experimental scheme to measure work and fluctuation relations in open quantum systems. However, the detection requires a finite size for the environment, which influences the system dynamics. This process cannot be modeled with the standard stochastic approaches. We develop a quantum jump model suitable for systems coupled to a finite-size environment. We use the method to study the common fluctuation relations and prove that they are satisfied.
\end{abstract}

DOI: 10.1103/PhysRevE.93.062106

\section{INTRODUCTION}

Rapid progress in the fabrication and manipulation of micro and nanoscale objects [1-4] has made it necessary to extend the concepts of thermodynamics to small systems, which by definition are not in the thermodynamic limit.

In such systems the extensive thermodynamic quantities, such as entropy, heat, and work, are not described by their average values alone but, due to fluctuations, they obey nontrivial probability distributions. Fortunately, it has been shown that, in many cases, the stochastic thermodynamic variables obey fluctuation relations [5-7] which often appear in the form of relations between exponential averages of the extensive variables.

While the two-measurement protocol of thermodynamic variables, especially work, is now well studied in closed quantum systems, there have been conceptual problems in open quantum systems [8-39]. To make a connection to classical stochastic thermodynamics, the quantum jump (QJ) method [40-44] has recently been used to study thermodynamics and fluctuation theorems in open quantum systems because it tries to mimic the trajectories realized in actual experiments [29-39]. The method unravels the master equation of the reduced density matrix as stochastic trajectories with environment-induced jumps between system states. The concepts of stochastic thermodynamics can be developed by associating a jump with heat exchange. However, there are several approximations that limit the generality of the QJ method. In particular, it is only applicable by assuming a memoryless or an infinitely large environment, i.e., an ideal heat bath whose state does not change during the drive.

Even within the QJ framework the issue of actually measuring the energy change in a driven open quantum system is nontrivial. It has been proposed by Pekola et al. [45] that this could be done by a so-called calorimetric measurement, where the immediate environment itself measures the energy change in the system. In Fig. 1, we show a schematic of such a setup in the case where there is a driven qubit coupled to a finite-size calorimeter and an ideal heat bath. The key point in the calorimetric measurement is that, in order to observe the energy changes of the system, the calorimeter must be finite, i.e., in contrast to the ideal bath it has to change its state when absorbing energy from the system [45-47].

The standard QJ approach is not applicable to the analysis of the calorimetric measurement setup, since the calorimeter is not ideal bath. In this paper, we develop a modified QJ model suitable for systems weakly coupled to a finite-size environment; called calorimeter from here on. In the model, a jump in the system changes both the state of the system and the state of the calorimeter. Due to the influence of the system on the calorimeter's evolution, the system evolution is no longer Markovian because the previous history of the system affects its future evolution via the state of the calorimeter. With the new method, we show that the common fluctuation relations are satisfied for the system-calorimeter composite. As a concrete example, we numerically study a sinusoidally driven qubit weakly coupled to the calorimeter which comprises harmonic oscillators with an energy gap equivalent to that of the qubit. The qubit and the calorimeter are initially thermalized with an ideal bath of inverse temperature $\beta$, as depicted in Fig. 1.

\section{THE STANDARD QUANTUM JUMP METHOD}

Let us first give a short introduction to the standard QJ method in the literature [40-44]. Instead of evolving the density matrix as done in the direct master equation

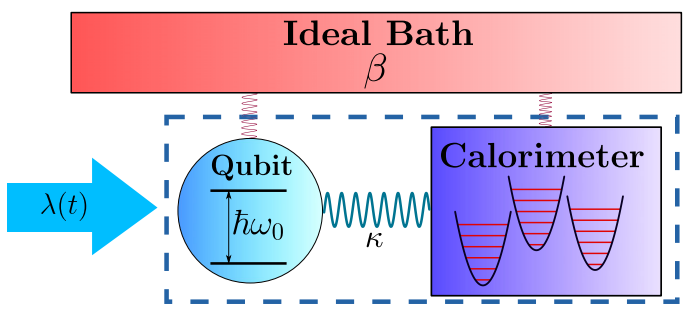

FIG. 1. Schematic drawing of the calorimetric measurement in a driven open quantum system. Here, a qubit is coupled to the calorimeter, which is described by finite number of harmonic oscillators with an energy gap equivalent to that of the qubit. The qubit and the calorimeter are initially thermalized with an ideal bath of inverse temperature $\beta$. The qubit is driven by a classical source $\lambda(t)$. 
calculations, the quantum jump method unravels the master equation into quantum trajectories with stochastically evolving wave functions. For a single trajectory, the probability for a jump between time $t$ and $t+\delta t$ is given by

$$
\delta p=\sum_{m} \delta p_{m}=\sum_{m} \delta t\left\langle\psi(t)\left|\hat{C}_{m}^{\dagger} \hat{C}_{m}\right| \psi(t)\right\rangle,
$$

for $\delta t \rightarrow 0$, where $|\psi(t)\rangle$ is the state of the system before the jump, $\delta p_{m}$ is the probability for a jump corresponding to a jump operator $\hat{C}_{m}=\sqrt{\Gamma_{m}} \hat{A}_{m}$. The matrix $\hat{A}_{m}$ gives the form of the jump and $\Gamma_{m}$ is the transition rate. If a jump corresponding to $\hat{C}_{m}$ occurs, the new state is given by $|\psi(t+\delta t)\rangle=\hat{C}_{m}|\psi(t)\rangle / \sqrt{\left(\delta p_{m} / \delta t\right)}$, where $\delta p_{m} / \delta t$ normalizes it. If no jumps occur during the time interval $[t, t+\delta t]$, the state evolution is not given by the system Hamiltonian $\hat{H}_{s}(t)$ alone but by the nonunitary Hamiltonian $\hat{H}(t)=\hat{H}_{s}(t)-\frac{i \hbar}{2} \sum_{m} \hat{C}_{m}^{\dagger} \hat{C}_{m}$, yielding

$$
|\psi(t+\delta t)\rangle=\frac{1}{\sqrt{1-\delta p}}\left(1-\frac{i}{\hbar} \hat{H}(t) \delta t\right)|\psi(t)\rangle .
$$

Although the jump operator can be time dependent, the past history of the trajectory, e.g., the number of jumps, does not affect the jump operators at all. As a consequence, the evolution of a stochastic trajectory depends only on the current state of the system $|\psi(t)\rangle$. This is a good approximation when the environment is ideal. However, such an environment makes the calorimetric measurement infeasible because the system evolution leaves no traces to the environment.

\section{QUANTUM JUMP METHOD WITH A FINITE ENVIRONMENT}

We now wish to extend the standard QJ method to the case corresponding to Fig. 1, where transitions in the system influence the state of the calorimeter, both initially thermalized with an ideal heat bath. We assume the calorimeter to be large enough to allow a semiclassical treatment such that there is an orthonormal eigenbasis where the calorimeter density matrix is diagonal. In practice, this basis will be the einselected basis of the calorimeter determined by the detector and the super bath [48]. We call these eigenbasis states microstates. We also assume the system-calorimeter coupling to be weak enough such that it can be neglected in the energy terms and modeled by stochastic jumps alone. We take into account only transitions that conserve the energy of the calorimeter-system composite.

To be precise, instead of using jump operators that only depend on the system degrees of freedom, we define new jump operators $\hat{D}_{m}=g_{m} \hat{A}_{m} \otimes \hat{B}_{m}$, where $\hat{A}_{m}$ causes a transition between system states and $\hat{B}_{m}$ between the calorimeter microstates defined above [49]. The coefficient $g_{m}$ is proportional to the coupling strength. We define the probability for a transition in the time interval $[t, t+\delta t]$ as

$$
\delta p=\sum_{m} \delta p_{m}=\sum_{m} \delta t \operatorname{Tr}_{s+c}\left\{\hat{D}_{m}^{\dagger} \hat{D}_{m} \hat{\sigma}_{s} \otimes \hat{\sigma}_{c}\right\},
$$

where $\delta p_{m}$ is the probability for a jump corresponding to the jump operator $\hat{D}_{m}, \hat{\sigma}_{s}(t)=|\psi(t)\rangle\langle\psi(t)|$ is the matrix form of the system state, $\hat{\sigma}_{c}(t)=|\Psi(t)\rangle\langle\Psi(t)|$ is the instantaneous calorimeter microstate, and the trace is over both the system $s$ and calorimeter $c$ degrees of freedom.

If a jump corresponding to $\hat{D}_{m}$ occurs, the new system and calorimeter states are given by $\hat{\sigma}_{s}(t+\delta t)=$ $\operatorname{Tr}_{c}\left\{\hat{D}_{m} \hat{\sigma}_{s} \otimes \hat{\sigma}_{c} \hat{D}_{m}^{\dagger}\right\} /\left(\delta p_{m} / \delta t\right)$ and $\hat{\sigma}_{c}(t+\delta t)=\operatorname{Tr}_{s}\left\{\hat{D}_{m} \hat{\sigma}_{s} \otimes\right.$ $\left.\hat{\sigma}_{c} \hat{D}_{m}^{\dagger}\right\} /\left(\delta p_{m} / \delta t\right)$.

If no jumps occur during the time interval $[t, t+\delta t]$, the time evolution is given by the nonunitary Hamiltonian

$$
\hat{H}(t)=\hat{H}_{s}(t)+\hat{H}_{c}-\frac{i \hbar}{2} \sum_{m} \hat{D}_{m}^{\dagger} \hat{D}_{m},
$$

where $\hat{H}_{s}$ and $\hat{H}_{c}$ are the system and calorimeter Hamiltonians, respectively. The new system state $\hat{\sigma}_{s}(t+\delta t)=$ $\operatorname{Tr}_{c}\left\{\hat{U}(t+\delta t, t) \hat{\sigma}_{s} \otimes \hat{\sigma}_{c} \hat{U}^{\dagger}(t+\delta t, t)\right\} /(1-\delta p)+O\left(d t^{2}\right)$, where $\hat{U}(t+\delta t, t)=1-\frac{i}{\hbar} \hat{H}(t) \delta t$. Similarly, the new calorimeter state $\hat{\sigma}_{c}(t+\delta t)=\operatorname{Tr}_{s}\left\{\hat{U}(t+\delta t, t) \hat{\sigma}_{s} \otimes \hat{\sigma}_{c} \hat{U}^{\dagger}(t+\delta t, t)\right\} /(1-$ $\delta p)+O\left(d t^{2}\right)$, which gives $\hat{\sigma}_{c}(t+\delta t)=\hat{\sigma}_{c}(t)$ because we assumed that $\hat{\sigma}_{c}(t)$ is in a microstate. As a consequence, it is sufficient to focus in detail only on the system dynamics, where the calorimeter's state only affects the transition rates.

\section{FLUCTUATION RELATIONS}

For studying stochastic thermodynamics and the associated fluctuation relations with this method, we focus on a generic two-level system (qubit) with $\hat{H}_{0}=\hbar \omega_{0} \hat{a}^{\dagger} \hat{a}$ that is weakly driven by a classical source $V(t)=\lambda(t)\left(\hat{a}+\hat{a}^{\dagger}\right)$, where $\hat{a}$ and $\hat{a}^{\dagger}$ are the annihilation and creation operators in the undriven basis. The system Hamiltonian is then given by $\hat{H}_{s}(t)=\hat{H}_{0}+\hat{V}(t)$. The qubit is coupled to the calorimeter by $\hat{V}_{N}(t)=\kappa \sum_{m}\left(\hat{a}^{\dagger} \hat{b}_{m}+\hat{a} \hat{b}_{m}^{\dagger}\right)$, where the coupling strength $\kappa$ is real and the operators $\hat{b}_{m}$ depend on the exact form of the calorimeter. The calorimeter Hamiltonian is given by $\hat{H}_{c}=\sum_{n} \epsilon_{n} \hat{d}_{n}^{\dagger} \hat{d}_{n}$. For a bosonic calorimeter $\hat{d}_{n}$ and $\hat{d}_{n}^{\dagger}$ are the annihilation and creation operators associated with energy $\epsilon_{n}$ and the operators $\hat{b}_{m}$ form a set of all the annihilation operators associated with energy $\hbar \omega_{0}$, i.e., $\sum_{m} \hat{b}_{m}=\sum_{n} \hat{d}_{n} \delta_{\epsilon_{n}}, \hbar \omega_{0}[50]$. Before and after the driving protocol, both the qubit and the calorimeter states are measured by monitoring the calorimeter state only. The qubit state can be indirectly determined from the previous jump before the drive and from the next jump after the drive in the calorimeter. However, this calorimetric monitoring is equivalent to performing the measurements by using the two-measurement protocol for both the qubit and the calorimeter, as shown in Appendix A.

The total system is initially prepared such that both the qubit and the calorimeter start as a pure state given by the joint probability $P\left[i, \Psi_{0}\right]$, where $|i\rangle$ and $\left|\Psi_{0}\right\rangle$ are the initial qubit and calorimeter states, respectively. Similar to the standard perturbative treatment [51], we define the jump operators in the undriven basis, i.e., $\hat{D}_{\uparrow, m}=g_{m} \hat{a}^{\dagger} \otimes \hat{b}_{m}$ and $\hat{D}_{\downarrow, m}=g_{m} \hat{a} \otimes \hat{b}_{m}^{\dagger}$, where the coefficient $g_{m} \propto \kappa$. Due to the energy change with the qubit, the calorimeter can jump to a different microstate such that the energy difference of the microstates corresponds to the energy change in the qubit. If the calorimeter is assumed to stay in the same microstate between the jumps, we can write the calorimeter traced jump 
operators [52] as $\hat{C}_{\uparrow, m}\left(\Psi_{k}\right)=\sqrt{\Gamma_{\uparrow, m}\left(\Psi_{k}\right)} \hat{a}^{\dagger}$ and $\hat{C}_{\downarrow, m}\left(\Psi_{k}\right)=$ $\sqrt{\Gamma_{\downarrow, m}\left(\Psi_{k}\right)} \hat{a}$, where the transition rates are

$$
\begin{aligned}
& \Gamma_{\uparrow, m}\left(\Psi_{k}\right)=\left|g_{k}\right|^{2} \operatorname{Tr}_{c}\left\{\hat{b}_{m} \hat{\sigma}_{c}\left(\Psi_{k}\right) \hat{b}_{m}^{\dagger}\right\}, \\
& \Gamma_{\downarrow, m}\left(\Psi_{k}\right)=\left|g_{k}\right|^{2} \operatorname{Tr}_{c}\left\{\hat{b}_{m}^{\dagger} \hat{\sigma}_{c}\left(\Psi_{k}\right) \hat{b}_{m}\right\},
\end{aligned}
$$

with $\hat{\sigma}_{c}\left(\Psi_{k}\right)=\left|\Psi_{k}\right\rangle\left\langle\Psi_{k}\right|$. If a jump caused by $\hat{C}_{\downarrow, m}\left(\Psi_{k}\right)$ occurs, the new calorimeter state is $\left|\Psi_{k+1}\right\rangle=\hat{b}_{m}^{\dagger}\left|\Psi_{k}\right\rangle /|| \hat{b}_{m}^{\dagger}\left|\Psi_{k}\right\rangle||$. According to stochastic thermodynamics [53,54], the entropy production associated with a jump is defined as the logarithmic ratio of the forward and backward transition rates. Due to the symmetry $\Gamma_{\downarrow, m}\left(\Psi_{k}\right)=\Gamma_{\uparrow, m}\left(\Psi_{k+1}\right)$, this entropy production is always zero and the total entropy production of a trajectory depends only on the initial and final states of the qubit and the calorimeter, i.e.,

$$
\Delta S_{T}=\ln \left\{P\left[i, \Psi_{0}\right] / \bar{P}\left[f, \Psi_{N}\right]\right\},
$$

where $\bar{P}\left[f, \Psi_{N}\right]$ is the probability to start a reversed trajectory with the final qubit state $|f\rangle$ and the final calorimeter state $\left|\Psi_{N}\right\rangle$ of the forward trajectory. The entropy production satisfies the fluctuation theorem (see Appendix B for details):

$$
\left\langle e^{-\Delta S_{T}}\right\rangle=1,
$$

where the average is over all the forward trajectories. If the initial probability distribution of the forward trajectory follows the canonical ensemble in equilibrium with the ideal heat bath, the probability distribution of the reversed trajectories can be chosen to follow a canonical ensemble with the same temperature. By defining the work $W$ associated with a single trajectory to be the energy difference between the final and initial states of the qubit-calorimeter composite, Eq. (7) gives the Jarzynski equality for work as

$$
\left\langle e^{-\beta W}\right\rangle=e^{-\beta \Delta F}
$$

where $\Delta F$ is the free-energy difference between the final and initial states.

The results above were derived by assuming that the calorimeter stays in the same microstate until the next jump. However, in many systems such as in electronic devices [55,56], the relaxation rate inside the calorimeter is the fastest timescale. Consequently, the calorimeter does not stay in a single microstate between jumps but shifts quickly between the microstates that correspond to the same energy. We can still calculate the qubit dynamics with Eqs. (2) and (3) by using an averaged calorimeter state instead of a single calorimeter microstate. According to the microcanonical ensemble, the averaged calorimeter state $\hat{\sigma}_{c}(E)=[1 / N(E)] \sum_{\Psi}|\Psi\rangle\langle\Psi| \delta_{E_{\Psi}, E}$, where the sum is over all the microstates, $N(E)$ is the number of microstates with energy $E$, and $E_{\Psi}$ is the energy of microstate $|\Psi\rangle$. Let us call this state a macrostate. We assume that the calorimeter reaches the macrostate instantaneously after a jump. The probability to start with the qubit state $|i\rangle$ and the calorimeter macrostate of energy $E_{0}$ is given by $P\left[i, E_{0}\right]$. Due to the energy change with the qubit, the calorimeter can jump to another macrostate. Because the calorimeter reaches the macrostate immediately after a jump, we can sum over all the transition rates corresponding the same energy change. The resulting total transition rates are given by

$$
\begin{aligned}
& \Gamma_{\downarrow}(E)=\frac{1}{N(E)} \sum_{m, \Psi} \Gamma_{\downarrow, m}(\Psi) \delta_{E_{\Psi}, E}, \\
& \Gamma_{\uparrow}(E)=\frac{1}{N(E)} \sum_{m, \Psi} \Gamma_{\uparrow, m}(\Psi) \delta_{E_{\Psi}, E},
\end{aligned}
$$

and they satisfy the detailed balance condition

$$
\Gamma_{\downarrow}\left(E-\hbar \omega_{0}\right) / \Gamma_{\uparrow}(E)=N(E) / N\left(E-\hbar \omega_{0}\right),
$$

which resembles the fluctuation relation derived for microcanonical ensembles $[57,58]$. The entropy production of a jump up with the calorimeter energy $E$ is given by

$$
\Delta s_{\uparrow}(E)=-\ln \left(\frac{\Gamma_{\downarrow}\left(E-\hbar \omega_{0}\right)}{\Gamma_{\uparrow}(E)}\right)=\ln \left(\frac{N\left(E-\hbar \omega_{0}\right)}{N(E)}\right),
$$

which gives a natural interpretation of the entropy production as the Boltzmann entropy change of the calorimeter. The entropy productions of up and down jumps are related by $\Delta s_{\uparrow}(E)=-\Delta s_{\downarrow}\left(E-\hbar \omega_{0}\right)$. The total entropy production is then given by

$$
\Delta S_{T}=\ln \left\{P\left[i, E_{0}\right] / \bar{P}\left[f, E_{N}\right]\right\}+\sum_{i=1}^{N} \Delta s_{\chi_{i}}\left(E_{i-1}\right),
$$

where $N$ is the number of jumps, $E_{i}$ is the calorimeter energy after the $i$ th jump, $\chi_{i}=\uparrow / \downarrow$ is the direction of $i$ th jump, and $\bar{P}\left[f, E_{N}\right]$ is the probability to start a reversed trajectory with the forward trajectory's final qubit state $|f\rangle$ and calorimeter energy $E_{N}$. Because Eq. (7) still holds, we recover the Jarzynski equality if we start from the canonical ensemble.

\section{NUMERICAL RESULTS}

To illustrate the method, we have done numerical simulations of the coupled qubit-calorimeter composite, where the calorimeter is described by $n$ quantum harmonic oscillators, with an energy gap equivalent to that of the qubit $\hbar \omega_{0}$. The harmonic oscillators can be noninteracting or they can interact fast enough such that the calorimeter can be assumed to reach the macrostate instantaneously after a jump. In both cases, the transition rates are the same and depend only on the calorimeter energy:

$$
\Gamma_{\downarrow}(E)=|g|^{2}\left(E+n \hbar \omega_{0}\right), \quad \Gamma_{\uparrow}(E)=|g|^{2} E,
$$

where we have for convenience chosen $E=0$ when all the oscillators are in the ground state. Consequently, the qubit's evolution depends only on the energy of the calorimeter. Because the calorimeter energy does not change between jumps, we can calculate the qubit dynamics by using Eqs. (2) and (3) with environment traced jump operators $\hat{C}_{\downarrow}=\sqrt{\Gamma_{\downarrow}(E)} \hat{a}$ and $\hat{C}_{\uparrow}=\sqrt{\Gamma_{\uparrow}(E)} \hat{a}^{\dagger}$ [52]. The calculations are done in the interaction picture with respect to $\hat{H}_{0}+\hat{H}_{e}$.

In order to compare the results with differing numbers of oscillators, we use the value $|g|^{2}=0.025 /(n \hbar)$ in the simulations such that the total coupling strength remains the 


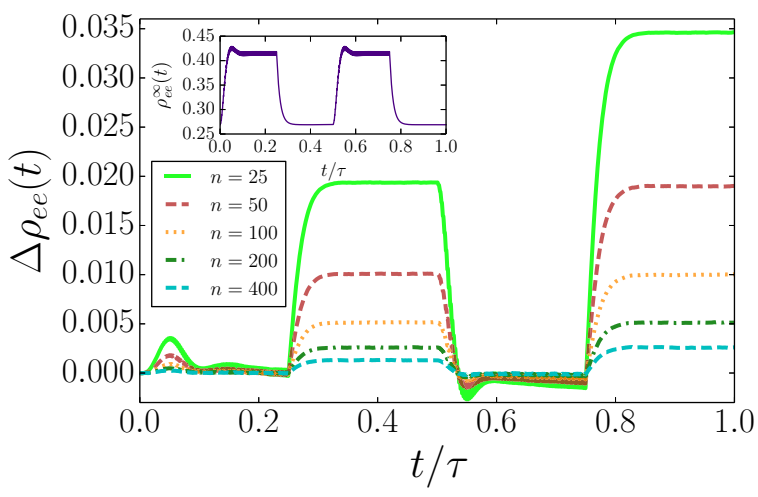

FIG. 2. The difference between the populations of the qubit excited state for $n$ and an infinite number of oscillators $\rho_{e}(t) \equiv$ $\rho_{e}^{n}(t)-\rho_{e}^{\infty}(t)$. The data are from numerical simulations of the coupled qubit-calorimeter system. The protocol ends at time $\tau$. The inset shows the excited state population when $n=\infty$. The drive is discretized by using $2 \times 10^{5}$ time steps and the number of trajectories is $10^{9}$. See text for the values of the other system parameters.

same. We start the qubit and the calorimeter from a canonical ensemble with respect to the inverse temperature $\beta=1 /\left(\hbar \omega_{0}\right)$. The qubit is driven sinusoidally with $\lambda(t)=0.05 \hbar \omega_{0} \sin \left(\omega_{0} t\right)$. The protocol consist of driving, no driving, driving, no driving parts; each lasting a time interval equal to 50 driving periods.

Figure 2 nicely illustrates the influence of the calorimeter's size on the qubit dynamics. Due to its finite size, the calorimeter is driven out of equilibrium. During the drive, the effect of the calorimeter on the qubit dynamics is suppressed by the drive since it is stronger than the qubit-calorimeter coupling. However, when the drive is stopped and the qubit equilibrates, the effect of the calorimeter becomes apparent. The drive causes the qubit to emit energy to the calorimeter. For a very large calorimeter (here the largest $n=400$ ), this additional energy is very small compared with the initial energy, which scales with size. However, for a smaller $n$ the change in the relative energy becomes more pronounced, as depicted in Fig. 3(b).

For the setup, the work is obtained as the energy difference between the final and initial states of the qubit plus the heat released to the calorimeter. This yields the same result as the two-measurement protocol for the composite. As shown in Figs. 3(a) and 3(c), the finite size of the calorimeter causes the work distribution to deviate from the infinite-size limit as overheating changes the transition rates. The transition rates are strongly influenced by the previous jumps for small values of $n$. This is illustrated in Fig. 3(c), where work values between $\pm 5 \hbar \omega_{0}$ are more probable for small $n$. Independent of $n$, the work distributions were found to be consistent with the Jarzynski equality within the statistical errors, as shown in Fig. 3(d).

\section{CONCLUSIONS}

Measurement and definition of work in driven open quantum systems poses an interesting theoretical and experimental challenge. In the present work, we consider the proposal of a calorimetric measurement that offers a simple and transparent way of measuring energy exchange between the system and the calorimeter. To theoretically analyze a calorimetric setup,
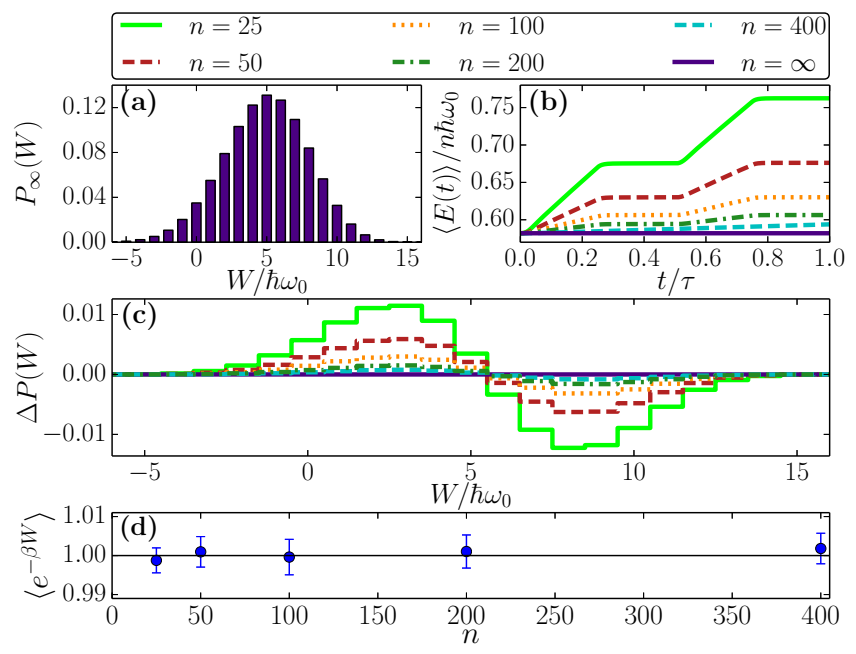

FIG. 3. Influence of finite-size calorimeter on the work and energy statistics when it consists of $n$ harmonic oscillators. (a) Probability distribution of work in the case of $n=\infty, P_{\infty}(W)$. (b) Time evolution of the average calorimeter energy for different values of $n$. (c) Deviation of the probability distribution of work $\Delta P(W) \equiv P_{n}(W)-P_{\infty}(W)$. (d) Numerical verification of the Jarzynski equality. The error bars are the standard deviation multiplied by 1.96 and correspond to a $95 \%$ confidence interval. The parameters are the same as in Fig. 2.

we developed a modified QJ model suitable for systems with a finite-size environment. We show that, due to the finite size, the calorimeter is driven out of equilibrium, leading to changes in the reduced system's dynamics and in the work statistics. These changes cannot be modelled with the standard QJ method, which assumes that the whole environment is an ideal heat bath. With the model, we have analytically and numerically shown that the standard fluctuation relations are still valid and remain unaltered by the finite size of the calorimeter. This is in contrast with Ref. [55], where the transition rates violate the detailed balance condition of Eq. (11) (see Appendix C).

\section{ACKNOWLEDGMENTS}

We wish to thank Jukka Pekola for suggesting this problem to us, and for useful discussions. We wish also to thank $\mathrm{C}$. Jarzynski, P. Muratore-Ginanneschi, and K. Schwieger for useful discussions and comments. This work was supported in part by the Väisälä Foundation and the Academy of Finland through its Centres of Excellence Programme (2015-2017) under project numbers 251748 and 284621. The numerical calculations were performed using computer resources of the Aalto University School of Science "Science-IT" project.

\section{APPENDIX A: EQUIVALENCE BETWEEN CALORIMETRIC MEASUREMENT AND TWO-MEASUREMENT PROTOCOL}

Let us assume that, after the drive, the qubit is in a superposition state $|\psi\rangle=\alpha_{0}|0\rangle+\alpha_{1}|1\rangle$, where $|0\rangle$ and $|1\rangle$ are the ground and excited states of the undriven qubit, respectively. The calorimeter is assumed to have energy $E$. A double projection measurement of both the qubit and the 
calorimeter then gives $E$ with probability $P(E)=\left|\alpha_{0}\right|^{2}$ and $E+\hbar \omega_{0}$ with probability $P\left(E+\hbar \omega_{0}\right)=\left|\alpha_{1}\right|^{2}=1-\left|\alpha_{0}\right|^{2}$.

In the calorimetric measurement, the qubit state is measured by waiting for the qubit to collapse to an eigenstate due to a jump. Let us first study the case where the calorimeter is in a single microstate $\left|\Psi_{k}\right\rangle$ and does not change between jumps. In this case, we can use the calorimeter traced jump operators $\hat{C}_{\uparrow, m}\left(\Psi_{k}\right)=\sqrt{\Gamma_{\uparrow, m}\left(\Psi_{k}\right)} \hat{a}^{\dagger}$ and $\hat{C}_{\downarrow, m}\left(\Psi_{k}\right)=\sqrt{\Gamma_{\downarrow, m}\left(\Psi_{k}\right)} \hat{a}$. The probability that the first jump after the drive is caused by jump operator $\hat{C}_{\downarrow, m}$ takes the form

$$
\begin{aligned}
P_{\downarrow, m}= & \int_{0}^{\infty} d t \Gamma_{\downarrow, m}\left(\Psi_{k}\right) \\
& \times\left|\left\langle 1\left|e^{-\frac{1}{2} \sum_{n}\left[\Gamma_{\downarrow, n}\left(\Psi_{k}\right)|1\rangle\left\langle 1\left|+\Gamma_{\uparrow, n}\left(\Psi_{k}\right)\right| 0\right\rangle\langle 0|\right] t}\right| \psi\right\rangle\right|^{2} \\
= & \int_{0}^{\infty} d t \Gamma_{\downarrow, m}\left(\Psi_{k}\right)\left|\alpha_{1}\right|^{2} e^{-\sum_{n} \Gamma_{\downarrow, n}\left(\Psi_{k}\right) t} \\
= & \frac{\left|\alpha_{1}\right|^{2} \Gamma_{\downarrow, m}\left(\Psi_{k}\right)}{\sum_{n} \Gamma_{\downarrow, n}\left(\Psi_{k}\right)} .
\end{aligned}
$$

The probability that the first jump after the drive is a jump down is obtained by summing over all $m$,

$$
P_{\downarrow}=\sum_{m} P_{\downarrow, m}=\left|\alpha_{1}\right|^{2} .
$$

In the case of a jump down, the qubit is known to be in the ground state after the jump and thus the total energy measured is simply the calorimeter energy after the jump, $E+\hbar \omega_{0}$. Similarly, the probability that the first jump after the drive is a jump up takes the form

$$
\begin{aligned}
P_{\uparrow}= & \sum_{m} \int_{0}^{\infty} d t \Gamma_{\uparrow, m}\left(\Psi_{k}\right) \\
& \times\left|\left\langle 0\left|e^{-\frac{1}{2} \sum_{n}\left[\Gamma_{\downarrow, n}\left(\Psi_{k}\right)|1\rangle\left\langle 1\left|+\Gamma_{\uparrow, n}\left(\Psi_{k}\right)\right| 0\right\rangle\langle 0|\right] t}\right| \psi\right\rangle\right|^{2} \\
= & \left|\alpha_{0}\right|^{2},
\end{aligned}
$$

giving total energy $E$. Thus, both measurement schemes produce equivalent energy distributions.

In the case that the calorimeter reaches the macrostate immediately after a jump, the calculation is similar with only two jump operators $\hat{C}_{\uparrow}(E)=\sqrt{\Gamma_{\uparrow}(E)} \hat{a}^{\dagger}$ and $\hat{C}_{\downarrow}(E)=$ $\sqrt{\Gamma_{\downarrow, m}(E)} \hat{a}$.

\section{APPENDIX B: FLUCTUATION THEOREM FOR QUANTUM JUMP MODEL WITH FINITE-SIZE ENVIRONMENT}

Let us consider an open quantum system coupled to a calorimeter through dissipative channels described by jump operators $\hat{D}_{m}=g_{m} \hat{A}_{m} \otimes \hat{B}_{m}$, where $\hat{A}_{m}$ and $\hat{B}_{m}$ depend on the system and calorimeter degrees of freedom, respectively, and $g_{m}$ is the coupling strength. We also assume that the jump operators follow detailed balance such that, for every $\hat{D}_{m}$, there is $\hat{D}_{n}$ such that $\hat{A}_{n}=\hat{A}_{m}^{\dagger}$ and $\hat{B}_{n}=\hat{B}_{m}^{\dagger}$. Let us first study the case where the calorimeter is in a single microstate $\left|\Psi_{k}\right\rangle$ and does not change between the jumps. In this case, we can use the calorimeter traced jump operators $\hat{C}_{m}=\sqrt{\Gamma_{m}\left(\Psi_{k}\right)} \hat{A}_{m}$ that are defined such that $\operatorname{Tr}_{s}\left\{\hat{C}_{m} \hat{\sigma}_{s} \hat{C}_{m}^{\dagger}\right\}=\operatorname{Tr}_{s+c}\left\{\hat{D}_{m} \hat{\sigma}_{s} \otimes\right.$ $\left.\left|\Psi_{k}\right\rangle\left\langle\Psi_{k}\right| \hat{D}_{m}^{\dagger}\right\}$, where $\hat{\sigma}_{s}$ is the matrix form of the system state, $\operatorname{Tr}_{\mathrm{s}}$ denotes trace over the system degrees of freedom, and $\operatorname{Tr}_{\mathrm{s}+\mathrm{c}}$ denotes the trace over both the system and calorimeter degrees of freedom. Due to the system-calorimeter energy change, the energy of the calorimeter evolves stochastically. The probability to traverse a single $N$-jump QJ trajectory is given by

$$
\begin{aligned}
& P_{Q J}\left[i, f, \Psi_{0}, \Psi_{N},\left\{\hat{C}_{m_{k}}\right\}_{k=1}^{N},\left\{t_{k}\right\}_{k=1}^{N}\right] \\
& \quad=P\left[i, \Psi_{0}\right]\left[\prod_{k=1}^{N} p^{0}\left(t_{k}, t_{k-1}\right) p_{m_{k}}\left(t_{k}\right)\right] p^{0}\left(\tau, t_{N}\right) P_{f}\left[f, \Psi_{N}\right],
\end{aligned}
$$

where the protocol starts at time $t_{0}=0$ and ends at time $\tau$, $P\left[i, \Psi_{0}\right]$ is the probability to start with the system state $|i\rangle$ and the calorimeter state $\left|\Psi_{0}\right\rangle, p_{m_{k}}\left(t_{k}\right)$ is the probability for a jump to occur along the $m_{k}$ th channel during $\left[t_{k}, t_{k}+\delta t\right]$, and $p^{0}\left(t_{k+1}, t_{k}\right)$ is the probability of no jump during the timeinterval $\left[t_{k}, t_{k+1}\right]$, and $P_{f}\left[f, \Psi_{N}\right]$ is the probability to measure the system state $|f\rangle$ and the calorimeter state $\left|\Psi_{N}\right\rangle$ at the end of the protocol. Because the jump probabilities can be calculated simply by using the calorimeter traced jump operators, we can use the results derived for time-dependent jump operators [38], yielding

$$
\begin{aligned}
P_{Q J} & {\left[i, f, \Psi_{0}, \Psi_{N},\left\{\hat{C}_{m_{k}}\right\}_{k=1}^{N},\left\{t_{k}\right\}_{k=1}^{N}\right] } \\
= & (\delta t)^{N} P\left[i, \Psi_{0}\right]\left[\prod_{k=1}^{N} \Gamma_{m_{k}}\left(\Psi_{k-1}\right)\right] \\
& \times\left|\left\langle f\left|\hat{U}_{\mathrm{eff}}\left(\tau, t_{N}\right)\left[\prod_{k=1}^{N} \hat{A}_{m_{N+1-k}} \hat{U}_{\mathrm{eff}}\left(t_{N+1-k}, t_{N-k}\right)\right]\right| i\right\rangle\right|^{2},
\end{aligned}
$$

where $\left|\Psi_{k}\right\rangle$ is the calorimeter state after the $k$ th jump and the no-jump evolution is given by

$$
\hat{U}_{\text {eff }}\left(t_{k+1}, t_{k}\right)=\mathcal{T} e^{-\frac{i}{\hbar}\left[\int_{t_{k}}^{t_{k}+1} \hat{H}_{s}(t)-i \frac{\hbar}{2} \sum_{i} \Gamma_{i}\left(\Psi_{k}\right) \hat{A}_{i}^{\dagger} \hat{A}_{i} d t\right]},
$$

where $\hat{H}_{s}(t)$ is the system Hamiltonian and $\mathcal{T}$ is the timeordering operator.

We can formulate a time-reversed counterpart for the forward trajectory of Eq. (B2). In the time-reversed trajectory, we measure system state $|f\rangle$ and calorimeter state $\left|\Psi_{N}\right\rangle$ at the beginning $(\bar{t}=0)$ and states $|i\rangle$ and $\left|\Psi_{0}\right\rangle$ at the end $(\bar{t}=\tau)$. In the time-reversed trajectory, all the jumps are reversed and they happen in reverse order, i.e., a jump caused by $\hat{C}_{\bar{m}_{k}}$ occurs at time $\bar{t}=t_{f}-t_{k}$, where the index $\bar{m}_{k}$ is related to the forward index $m_{k}$ such that $\hat{A}_{\bar{m}_{k}}=\hat{A}_{m_{k}}^{\dagger}$ and $\hat{B}_{\bar{m}_{k}}=\hat{B}_{m_{k}}^{\dagger}$. By demanding that the time-reversed no-jump evolution between jumps is given by $\hat{U}_{\mathrm{eff}}^{\dagger}\left(t_{i+1}, t_{i}\right)$, the probability for the reverse QJ trajectory can then be written as

$$
\begin{aligned}
\bar{P}_{Q J} & {\left[f, i, \Psi_{N}, \Psi_{0},\left\{\hat{C}_{\bar{m}_{k}}\right\}_{k=1}^{N},\left\{\bar{t}_{k}\right\}_{k=1}^{N}\right] } \\
= & (\delta t)^{N} \bar{P}\left[f, \Psi_{N}\right]\left[\prod_{k=1}^{N} \Gamma_{\bar{m}_{k}}\left(\Psi_{k}\right)\right] \\
& \times\left|\left\langle i\left|\left[\prod_{k=1}^{N} \hat{U}_{\mathrm{eff}}^{\dagger}\left(t_{k}, t_{k-1}\right) \hat{A}_{m_{k}}^{\dagger}\right] \hat{U}_{\mathrm{eff}}^{\dagger}\left(\tau, t_{N}\right)\right| f\right\rangle\right|^{2},
\end{aligned}
$$

where $\bar{P}\left[f, \Psi_{N}\right]$ is the probability to start a reversed trajectory with the system state $|f\rangle$ and the calorimeter state $\left|\Psi_{N}\right\rangle$. The 
ratio of the forward and reversed trajectory probabilities is of the form

$$
\begin{aligned}
\Delta S_{T} & {\left[i, f, \Psi_{0}, \Psi_{N},\left\{\hat{C}_{m_{k}}\right\}_{k=1}^{N},\left\{t_{k}\right\}_{k=1}^{N}\right] } \\
& =\ln \left[\frac{P_{Q J}\left[i, f, \Psi_{0}, \Psi_{N},\left\{\hat{C}_{m_{k}}\right\}_{k=1}^{N},\left\{t_{k}\right\}_{k=1}^{N}\right]}{\bar{P}_{Q J}\left[f, i, \Psi_{N}, \Psi_{0},\left\{\hat{C}_{\bar{m}_{k}}\right\}_{k=1}^{N},\left\{\bar{t}_{k}\right\}_{k=1}^{N}\right]}\right] \\
& =\ln \left\{\frac{P\left[i, \Psi_{0}\right]}{\bar{P}\left[f, \Psi_{N}\right]}\right\}+\ln \left[\prod_{k=1}^{N} \frac{\Gamma_{m_{k}}\left(\Psi_{k-1}\right)}{\Gamma_{\bar{m}_{k}}\left(\Psi_{k}\right)}\right] .
\end{aligned}
$$

We denote this term as the total entropy production of the model. As the reversed trajectories' probabilities sum up to unity, it can be straightforwardly shown that

$$
\left\langle e^{-\Delta S_{T}}\right\rangle=1,
$$

where the average is over all the forward trajectories.

As discussed in the main text, the transition rates satisfy the condition $\Gamma_{\bar{m}_{k}}\left(\Psi_{k}\right)=\Gamma_{m_{k}}\left(\Psi_{k-1}\right)$ when the calorimeter is assumed to be in a single microstate. As a consequence, the second term of Eq. (B5) is zero and the entropy production depends only on the forward and reversed trajectory initial probability distributions. If the initial probability distribution of the forward trajectory follows canonical ensemble, the probability distribution of the reversed trajectories can be chosen to follow a canonical ensemble with the same temperature. In this case, the total entropy production becomes equivalent with the energy difference between the final and initial state of the total system. By defining the work of a single trajectory as the energy difference between the final and initial state of the total system, Eq. (B6) gives the Jarzynski equality.

If the relaxation rate inside the calorimeter is the fastest timescale, then the calorimeter does not stay in a single microstate between jumps but shifts quickly between the microstates $\left|\Psi_{k}\right\rangle$ that correspond to the same energy. We can still derive a theorem similar to Eq. (B5) with $\Psi_{k}$ denoting calorimeter energies instead of states. The transition rates are then given by Eqs. (9) and (14) of the main text. In this case, the product of the transition rate ratio gives $N\left(E_{0}\right) / N\left(E_{N}\right)$ in Eq. (B5), where $E_{0}$ and $E_{N}$ are the initial and final energies of the calorimeter, respectively, and $N(E)$ denotes the number of microstates corresponding to energy $E$. If both the forward and backward process start from the canonical ensemble, i.e., $P\left[i, E_{0}\right]=N\left(E_{0}\right) e^{-\beta\left(\hbar \omega_{i}+E_{0}\right)} / Z$ and $\bar{P}\left[f, E_{N}\right]=N\left(E_{N}\right) e^{-\beta\left(\hbar \omega_{f}+E_{N}\right)} / Z^{\prime}$, we get again the
Jarzynski equality with $\Delta F=-(1 / \beta) \ln \left(Z^{\prime} / Z\right)$. Here, $\hbar \omega_{i}$ and $\hbar \omega_{f}$ denote the energies of system states $|i\rangle$ and $|f\rangle$, respectively.

\section{APPENDIX C: DETAILED BALANCE CONDITION FOR TRANSITION RATES}

When the energy fluctuations are small in the canonical ensemble, they are often approximated by a Gaussian distribution around the average energy $E_{0}$. If the heat capacity $C$ is constant, these energy fluctuations are often expressed as the effective temperature fluctuations $T=E / C$ in mesoscopic systems $[55,59,60]$. We will now show that the assumption of a constant heat capacity can lead to violation of the main text's Eq. (11) when temperature fluctuations $T$ are used together with the standard temperature-dependent transition rates

$$
\begin{gathered}
\Gamma_{\downarrow}\left(\hbar \omega_{0}, T(E)\right)=|g|^{2} /\left(1 \pm e^{-\hbar \omega_{0} /\left(k_{B} T\right)}\right), \\
\Gamma_{\uparrow}\left(\hbar \omega_{0}, T(E)\right)=|g|^{2} /\left(e^{\hbar \omega_{0} /\left(k_{B} T\right)} \pm 1\right),
\end{gathered}
$$

where $g$ is the coupling strength, $\hbar \omega_{0}$ is the energy gap of the qubit, $T(E)$ is the effective temperature corresponding to the calorimeter energy $E$, and $+(-)$ is used in the case fermionic (bosonic) transition rates. If a jump up occurs in the qubit, the energy of the calorimeter decreases by $\hbar \omega_{0}$ and consequently the effective temperature decreases by $\hbar \omega_{0} / C$.

For Gaussian energy fluctuations, the left-hand side of Eq. (11) simplifies to

$$
\frac{N(E)}{N\left(E-\hbar \omega_{0}\right)}=e^{\hbar \omega_{0}\left\{\beta+\left[\hbar \omega_{0}-2\left(E-E_{0}\right)\right] /\left(2 \sigma^{2}\right)\right\}},
$$

where $N(E)$ and $N\left(E-\hbar \omega_{0}\right)$ are the number of microstates corresponding to calorimeter energies $E$ and $E-\hbar \omega_{0}$, respectively, $\beta$ is the inverse temperature of the ideal bath that is used to thermalized both the qubit and the calorimeter, and $\sigma^{2}=C k_{B}^{-1} \beta^{-2}$ is the variance of the Gaussian calorimeter energy distribution. However, if now the transition rates of Eqs. (C1) and (C2) are used, the right-hand side of Eq. (11) does not agree with Eq. (C3) and thus the detailed balance condition is not satisfied. This explains the apparent violations of Jarzynski equality in Ref. [55], where similar types of transition rates were used with Gaussian effective temperature fluctuations and a constant heat capacity.
[1] S. Nakamura, Y. Yamauchi, M. Hashisaka, K. Chida, K. Kobayashi, T. Ono, R. Leturcq, K. Ensslin, K. Saito, Y. Utsumi et al., Phys. Rev. Lett. 104, 080602 (2010).

[2] B. Küng, C. Rössler, M. Beck, M. Marthaler, D. S. Golubev, Y. Utsumi, T. Ihn, and K. Ensslin, Phys. Rev. X 2, 011001 (2012).

[3] J. P. Pekola, Nat. Phys. 11, 118 (2015).

[4] J. Roßnagel, S. T. Dawkins, K. N. Tolazzi, O. Abah, E. Lutz, F. Schmidt-Kaler, and K. Singer, Science 352, 325 (2016).

[5] G. N. Bochkov and Y. E. Kuzovlev, Sov. Phys. JETP 45, 125 (1977).

[6] C. Jarzynski, Phys. Rev. Lett. 78, 2690 (1997).

[7] U. Seifert, Phys. Rev. Lett. 95, 040602 (2005).
[8] M. Esposito and S. Mukamel, Phys. Rev. E 73, 046129 (2006).

[9] M. Campisi, P. Talkner, and P. Hänggi, Phys. Rev. Lett. 102, 210401 (2009).

[10] S. Deffner and E. Lutz, Phys. Rev. Lett. 107, 140404 (2011).

[11] G. E. Crooks, J. Stat. Mech. (2008) P10023.

[12] R. Chetrite and K. Mallick, J. Stat. Phys. 148, 480 (2012).

[13] T. Albash, D. A. Lidar, M. Marvian, and P. Zanardi, Phys. Rev. E 88, 032146 (2013).

[14] M. Esposito, U. Harbola, and S. Mukamel, Rev. Mod. Phys. 81, 1665 (2009).

[15] M. Campisi, P. Hänggi, and P. Talkner, Rev. Mod. Phys. 83, 771 (2011). 
[16] A. E. Rastegin and K. Życzkowski, Phys. Rev. E 89, 012127 (2014).

[17] G. Watanabe, B. P. Venkatesh, P. Talkner, M. Campisi, and P. Hänggi, Phys. Rev. E 89, 032114 (2014).

[18] M. Silaev, T. T. Heikkilä, and P. Virtanen, Phys. Rev. E 90, 022103 (2014).

[19] S. Gasparinetti, P. Solinas, A. Braggio, and M. Sassetti, New J. Phys. 16, 115001 (2014).

[20] J. Salmilehto, P. Solinas, and M. Möttönen, Phys. Rev. E 89, 052128 (2014).

[21] J. Ankerhold and J. P. Pekola, Phys. Rev. B 90, 075421 (2014).

[22] C. Jarzynski, H. T. Quan, and S. Rahav, Phys. Rev. X 5, 031038 (2015).

[23] T. B. Batalhão, A. M. Souza, L. Mazzola, R. Auccaise, R. S. Sarthour, I. S. Oliveira, J. Goold, G. De Chiara, M. Paternostro, and R. M. Serra, Phys. Rev. Lett. 113, 140601 (2014).

[24] S. An, J.-N. Zhang, M. Um, D. Lv, Y. Lu, J. Zhang, Z.-Q. Yin, H. T. Quan, and K. Kim, Nat. Phys. 11, 193 (2015).

[25] S. Deffner and A. Saxena, Phys. Rev. Lett. 114, 150601 (2015).

[26] M. Carrega, P. Solinas, A. Braggio, M. Sassetti, and U. Weiss, New J. Phys. 17, 045030 (2015).

[27] R. Schmidt, M. F. Carusela, J. P. Pekola, S. Suomela, and J. Ankerhold, Phys. Rev. B 91, 224303 (2015).

[28] B. P. Venkatesh, G. Watanabe, and P. Talkner, New J. Phys. 17, 075018 (2015)

[29] J. M. Horowitz, Phys. Rev. E 85, 031110 (2012).

[30] F. W. J. Hekking and J. P. Pekola, Phys. Rev. Lett. 111, 093602 (2013).

[31] J. M. Horowitz and J. M. R. Parrondo, New J. Phys. 15, 085028 (2013).

[32] B. Leggio, A. Napoli, A. Messina, and H.-P. Breuer, Phys. Rev. A 88, 042111 (2013).

[33] F. Liu, Phys. Rev. E 89, 042122 (2014).

[34] S. Suomela, P. Solinas, J. P. Pekola, J. Ankerhold, and T. AlaNissila, Phys. Rev. B 90, 094304 (2014).

[35] J. Horowitz and T. Sagawa, J. Stat. Phys. 156, 55 (2014).

[36] F. Liu, Phys. Rev. E 93, 012127 (2016).

[37] J. P. Pekola, Y. Masuyama, Y. Nakamura, J. Bergli, and Y. M. Galperin, Phys. Rev. E 91, 062109 (2015).

[38] S. Suomela, J. Salmilehto, I. G. Savenko, T. Ala-Nissila, and M. Möttönen, Phys. Rev. E 91, 022126 (2015).
[39] G. Manzano, J. M. Horowitz, and J. M. R. Parrondo, Phys. Rev. E 92, 032129 (2015).

[40] J. Dalibard, Y. Castin, and K. Mølmer, Phys. Rev. Lett. 68, 580 (1992).

[41] R. Dum, P. Zoller, and H. Ritsch, Phys. Rev. A 45, 4879 (1992).

[42] C. W. Gardiner, A. S. Parkins, and P. Zoller, Phys. Rev. A 46, 4363 (1992).

[43] H. J. Carmichael, An Open Systems Approach to Quantum Optics (Springer, New York, 1993).

[44] M. B. Plenio and P. L. Knight, Rev. Mod. Phys. 70, 101 (1998).

[45] J. P. Pekola, P. Solinas, A. Shnirman, and D. V. Averin, New J. Phys. 15, 115006 (2013).

[46] S. Gasparinetti, K. L. Viisanen, O.-P. Saira, T. Faivre, M. Arzeo, M. Meschke, and J. P. Pekola, Phys. Rev. Appl. 3, 014007 (2015).

[47] K. L. Viisanen, S. Suomela, S. Gasparinetti, O.-P. Saira, J. Ankerhold, and J. P. Pekola, New J. Phys. 17, 055014 (2015).

[48] W. H. Zurek, Rev. Mod. Phys. 75, 715 (2003).

[49] That is, the calorimeter operators are of the form $\hat{B}_{m}=$ $\left|\Psi_{m}^{\prime}\right\rangle\left\langle\Psi_{m}\right|$, where $\left|\Psi_{m}\right\rangle$ and $\left|\Psi_{m}^{\prime}\right\rangle$ are calorimeter microstates.

[50] The analytical results here are also valid for a Fermi bath. In that case, $\hat{d}_{n}$ and $\hat{d}_{n}^{\dagger}$ are the Fermi operators and the operators $\hat{b}_{m}$ are transitions between calorimeter states that correspond $\hbar \omega_{0}$, i.e., $\sum_{m} \hat{b}_{m}=\sum_{n, l} \hat{d}_{n}^{\dagger} \hat{d}_{l} \delta_{\epsilon_{n}, \epsilon_{l}-\hbar \omega_{0}}$.

[51] A. Clerk, M. Devoret, S. Girvin, F. Marquardt, and R. Schoelkopf, Rev. Mod. Phys. 82, 1155 (2010).

[52] The calorimeter traced jump operators are defined such that $\operatorname{Tr}_{s}\left\{\hat{C}_{m} \hat{\sigma}_{s} \hat{C}_{m}^{\dagger}\right\}=\operatorname{Tr}_{s+c}\left\{\hat{D}_{m} \hat{\sigma}_{s} \otimes \hat{\sigma}_{c} \hat{D}_{m}^{\dagger}\right\}$.

[53] G. E. Crooks, Phys. Rev. E 60, 2721 (1999).

[54] J. M. Parrondo, J. M. Horowitz, and T. Sagawa, Nat. Phys. 11, 131 (2015).

[55] J. P. Pekola, A. Kutvonen, and T. Ala-Nissila, J. Stat. Mech. (2013) P02033.

[56] A. Kutvonen, T. Ala-Nissila, and J. Pekola, Phys. Rev. E 92, 012107 (2015).

[57] B. Cleuren, C. Van den Broeck, and R. Kawai, Phys. Rev. Lett. 96, 050601 (2006).

[58] Y. Subasi and C. Jarzynski, Phys. Rev. E 88, 042136 (2013).

[59] L. D. Landau and E. Lifshitz, Course Theor. Phys. 5, 468 (1980).

[60] T. T. Heikkilä and Y. V. Nazarov, Phys. Rev. Lett. 102, 130605 (2009). 